\title{
XIII. The anomalous dispersion of sodium vapour
}

\section{R.W. Wood}

To cite this article: R.W. Wood (1902) XIII. The anomalous dispersion of sodium vapour, Philosophical Magazine Series 6, 3:13, 128-144, DOI: 10.1080/14786440209462746

To link to this article: http://dx.doi.org/10.1080/14786440209462746

册 Published online: 09 Jun 2010.

Submit your article to this journal $\pi$

Џlll Article views: 3

Q View related articles ¿

4 Citing articles: 5 View citing articles $\square$ 
the first being due to primary radiation alone, that in the second to primary and secondary radiation.

The method employed in measuring the absorption of the primary rays was also a comparative one, and was practically identical with that of Rutherford *.

As yet the determinations have only been completed in the case of a single gas - sulphuretted hydrogen-in which the coefficient of absorption is comparatively large. In this gas, calling the ionization produced by the primary rays in a distance of $2.5 \mathrm{~cm}$. 100 , the ionization caused by the secondary rays from a freshly polished brass plate, $10 \mathrm{~cm}$. by $5 \mathrm{~cm}$. in area, amounted to 11 . The variation due to the absorption of $20 \mathrm{~cm}$. of sulphuretted hydrogen was found to be 3.9 per cent., giving as the value of $\lambda 1.9 \times 10^{-3}$.

Hence the ratio of the total ionization caused by the secondary radiation from a brass plate to the total ionization for the primary radiation is equal to

$$
\frac{11}{100} \times 2 \cdot 5 \times 1 \cdot 9 \times 10^{-3} \text {, }
$$

or $\frac{1}{1900}$ very nearly.

From this it follows on the foregoing assumptions that the total energy of the secondary radiation only amounts to about one two-thousandth part of the energy of the primary radiation.

The work here described was carried out at the Cavendish Laboratory at the suggestion of Professor J. J. Thomson, to whom I am glad to express my indebtedness.

Trinity College, Cambridge.

XIII. The Anomalous Dispersion of Sodium Vapour. By R. W. Wood, Professor of Physics in the University of Wisconsint.

$$
\text { [Plates II.-IV.] }
$$

GXPERIMENTAL proof of the dispersion formulæ of E Sellmeier, Helmboltz, and Ketteler has for the most part been based upon observations made upon substances in the solid state or in the state of solution, where the range of absorption extends over a considerable portion of the

* Phil. Mag. [5] xliii. pp. \$59-255 (1897).

+ Read before the Royal Society June 20, 1901. We are indebted to the Royal Society for the use of the stone for the plate and to the Author for defraying part of the expense of reproducing it. 
spectrum, and the molecular condition is in all probability vastly more complicated than in the gaseous state.

In liquid oxygen and in the salts of erbium and didymium we have the only examples, so far as I know, of substances other than gases which exhibit narrow absorption-bands, and in these cases the absorption is scarcely strong enough to influence the dispersion to any marked degree. A careful study of the dispersion of some substance with absorptionbands as narrow and strong as those of sodium vapour, would be of great value in aiding us to pass judgment on the various modifications of Sellmeier's original theory.

The anomalous dispersion of sodium vapour in the immediate vicinity of the $D$ lines was first observed by Kundt, and has since been studied by Becquerel and Julius, but none of these investigators has traced the effect over a range of the spectrum greater than two or three times the distance between the lines.

In a previous paper* I have shown that a spectrum closely resembling the bright-line spectrum of incandescent sodium can be produced under certain conditions by the anomalous refraction of white light in a non-homogeneous atmosphere of sodium vapour, in precisely the manner in which Julius very ingeniously suggests that it may occur in the reversing layer of the sun, giving rise to the phenomenon of the "flash spectrum." In view of the seeming importance of the subject in its bearing on the subject of the dynamics of dispersion, and its possible connexion with solar physics, I have undertaken to determine whether sufficiently accurate quantitative data can be obtained, to make a test of the dispersion formula, as applied to sodium vapour, possible.

Though the absolute values of the refractive indices which have been found are not as satisfactory as is desirable, very interesting relative values have been obtained, and, what is of especial interest, the dispersion has been traced and measured throughout the entire range of the visible spectrum, the refractive index for all waves of higher frequency than $\mathrm{D}_{2}$ being less than one, and for all waves of lower frequency than $D_{1}$ greater than one. In other words, I have been able to form a complete anomalous spectrum by means of sodium vapour, in which all of the colours between the extreme red and violet are present, with the exception of a range of perhaps twenty Angström units at the D lines.

Previous investigations have been made exclusively with * Phil. Mag. May 1901.

Phil. Mag. S. t. Vol. 3. No. 13. Jan. 1902. 
sodium flames, which have been made to assume a prismatic form. In view of the great uncertainty regarding the prism angle, density of the vapour, and its molecular condition, that is whether dissociated or not, it seemed advisable to employ non-luminous vapour, obtained by heating metallic sodium in some inert gas or in vacuo.

The first experiments were made with a large prism of cast iron, furnished with windows of mica or thin plate glass, in which the metal was beated in an atmosphere of hydrogen. Very beautiful results were at once obtained, but certain peculiarities of the vapour's action showed that the refraction was due chiefly to the action of a non-homogeneous medium, the planes of constant density being horizontal. Great trouble was had with the windows, which soon became covered with a white deposit, which cut off most of the light. It being apparent, however, that the oblique faces played but a very small part, the effect being due almost wholly to the variable density of the vapour, it seemed best to make the most of this circumstance, and dispense with the trouble entirely by removing the glass plates to such a distance from the heated vapour that no deposit took place. The arrangement finally adopted $\mathrm{u}$ as simply a tube of glass about $30 \mathrm{~cm}$. long, provided with plate-glass ends cemented on with sealing-wax. Hydrogen, dried by passage over calcium chloride, was conducted into and out of the tube by means of two fine glass tubes arranged as shown in fig. 1 .

Fig. 1.

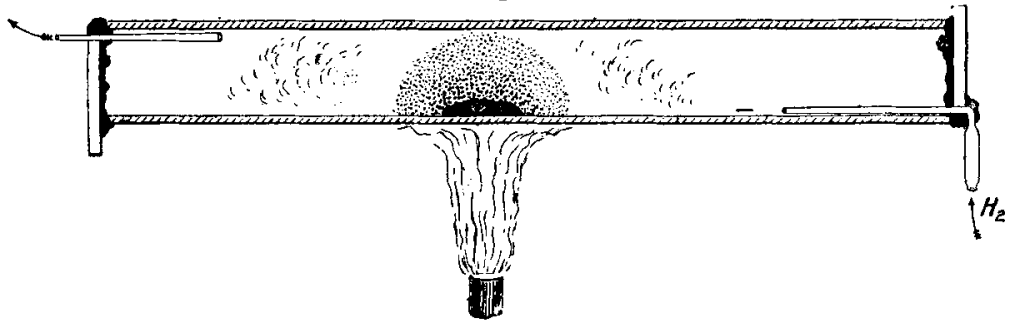

The diameter of the tubes should not be over $2 \mathrm{~mm}$, and they should lie close against the sides of the large tube in order not to cut off any of the light. The most suitable diameter for the large tube is $2 \mathrm{~cm}$. As the experiments to be described in this paper will, I feel sure, repay any one who takes the trouble to repeat them, and will be extremely useful in illustrating the subject of anomalous dispersion to small classes of advanced students, I shall describe in some 
detail the manner of preparing and using these dispersiontubes, which I have found most advantageous. The ends of the tubes are first warmed and thickly coated with sealingwax; one of the glass straws is then placed in position, and a small piece of plate glass, previously warmed, pressed against the wax, any crevices around the straw being closed with wax. The leading-in tube is next placed in position, and a piece of freshly-cut sodium (about $5 \mathrm{~mm}$. on a side) inserted. The other window is then cemented to the tube, and the current of hydrogen started as soon as possible. Some experience is necessary properly to regulate the hydrogen stream during the experiment. When the tube is first heated much white smoke forms. If a stream corresponding to about one bubble per second is allowed to flow, the smoke will usually clear up in a few minutes and give little trouble. The tube should be heated by means of a Bunsen burner turned down low, the tip of the flame playing against the bottom of the tube. If a sodium flame is placed behind the tube the formation of the vapour can be watched, for it appears almost jet-black a minst the flame, though quito colourless in white light. The behaviour of the vapour is somewhat peculiar. It grows out from the sodium globule as a dark atmosphere with a sharpiy defined surface, which clings to the globule with great tenacity. It resembles at first a thick growth of mould more than anything else that I can think of, and a sudden gust of hydrogen scarcely moves it at all. A wire pushed up through it drags a certain amount above the free surface in much the same manner as a stick pushed up through the surface of thick molasses would do. If the tube be inverted the black cloud chngs to the upper surface, behaving on the whole like a very viscous mass. It is even possible to dip some of it up on a wire,

These peculiar physical properties of the metallic vapour I have as yet only studied in a very superticial manner, and I mention them now, only because it appears to me that there is some connexion between them and the optical behaviour of the medium.

I am of the opinion that the apparent viscosity is an illusion, and that the sharply-defined surface is merely the boundary at which either condensation or chemical action (the hydrogen not being pure) is taking place. The process of dipping the vapour up on the wire might be explained by condensation on the wire followed by vaporization. A more careful study of the physical behaviour of the vapour will be made some time in the future.

The apparatus employed in the study of the dispersion K 2 
of the vapour was essentially identical with that used by Becquerel. The light of an arc limp was focussed on the horizontal slit of a collimator, after traversing which the parallel rays passed lengthwise through the di-persion-tube. A second lens brought them to a focus on the slit of a spectroscope, when the dispersion was to be studied by the method of crossed prisms, or in the focus of an eyepiece when the anomalous spectrum was to be viewed subjectively (fig. 2).

Fig. 2.

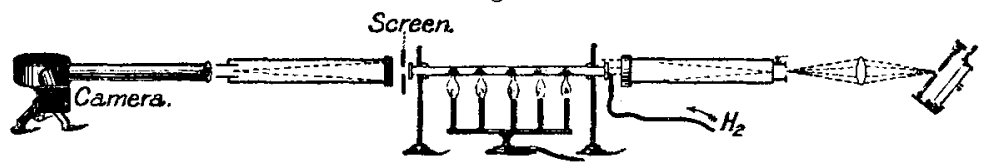

The first experiments were made by the method of crossed prisms, the spectrometer being furnished with a Rowlind plane grating, which showed the sodium lines widely separated. It was at once apparent that far better results could be obtained with the dispersion-tubes than had ever been observed with prismatic flames. The curved branches, of the diffraction-spectrum on each side of the $D$ lines were perfectly sharp and steady, and the dispersion could be traced to a considerable distance up and down the spectrum. On the slit of the spectrometer appeared, instead of the white image of the horizontal slit, a most beautiful anomalous spectrum, of great brilliancy and purity. The spectrometer was at once removed and an eyepiece put in its place, when a most superb spectrum revealed itself. The general appearance is shown in the coloured plate accompanying this raper, though it is quite impossible to represent by means of pigments the sparkling brilliancy of the colours.

Before discussing this spectrum in detail, it will be better to take up the results of this experiments made by the method of crossed prisms. On first heating the tube, the curvature of the spectrum between the $D$ lines as well as on each side is observed, the appearance being identical with that figured by Becquerel, but in a few seconds the vapour becomes so dense that total absorption of all the light between the lines occurs. Julius expresses the opinion that this disappearance of the light between the lines is only a result of the strong dispersion; that is, it is not absorbed but turned off to one side so that it does not enter the instrument. It appears to me, however, that this is not the case, for $I$ have ouserved the same effect under conditions where lateral deflexion seems quite out of the question. As I shall show 
later on, the breadth of the absorption-band is sometimes twenty times the width of the spectrum comprised within the $\mathrm{D}$ lines.

The oppositely-curved branches adjacent to the region of absorption grow out rapidly as the tube grows hotter, the ends finally passing out of the field of the instrument. A beautiful fluted absorption appears in the red and the greenishblue, which finally blots out a region in the blue almost entirely. Meanwhile the curvature of the spectrum increases in a most remarkable manner, and the entire red end is lifted ligh above the green-blue end. As the density of the vapour increases the red gradually fades away, leaving only the yellow and green and the remote blue and violet, the curvature increasing all the while. The fluted or channelled spectrum was described by Roscoe and Schuster about twenty-tive years ago, but so far as I know no work has been done on it since. I have recently secured excellent photographs of it with a Rowland concave grating, from the extreme red to the violet, and find that it is much more extensive than has been supposed, for the flutings run right up to the absorption-band at the D lines on both sides, thongh they are very faint on the side of shorter wavelengths. This spectrum will be described in a subsequent paper.

Very satisfactory photographs of the dispersed gratingspectrum were secured, some of which are reproduced. It was found impossible to maintain a sufficiently uniform density, at the low temperature, for a sufficient length of time to enable a negative to be secured showing the appearance before the light between the $\mathrm{D}$ lines vanished. I therefore went back to the old plan of using a prismatic flame. After some experimenting it was found that the most satisfactory tlame was secured by passing hydrogen through a tube containing metallic sodium and strongly $\mathrm{h}$ sated, and burning the gas at a flat jet piece made of platinum foil. An exceedingly dense and very uniform sodium flume is obtiined in this way, which can be maintained almost indefinitely. The arrangement of the lamp is shown in fig. 3 (p. 134), the diagram requiring no description.

With this flame as a dispersing piece the photographs shown in Plate III. fig. 8 were obtained. In comparison with some of the other photographs this example is very poor, and I have included it merely to make the set complete. An exposure of about ten minutes was necessary, with an "Erythro" plate. Fig. 9 was taken with one of the dispersion-tubes, and shows what may be called the second 
stage. After the exposure was over, but before the plate was removed, a small sodium flame was placed before the slit of the spectrometer, and the sodium lines impressed on the plate in their proper position, to serve as a reference. For securing photographs of the dispersiun resulting from still denser vapour more light was desirable, and a small prism was accordingly substituted for the grating. This prism barely resolved the sodium lines in the negatives, but gave

Fig. 3.

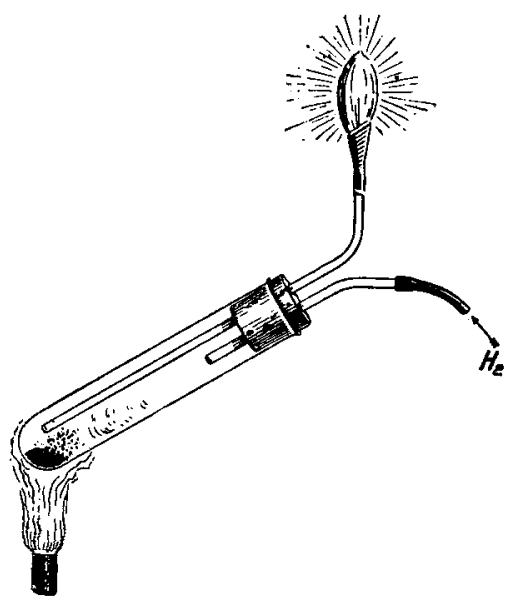

excellent pictures of the spectrum under strong sodium dispersion. Pl. III. figs. 10 and 11 were obtained in this way. In the latter the flutings in the red and green are discernible, though not very sharp.

As much of the fine detail is always lost in reproduction I have marked with arrows on fig. 11 the extreme points to which the curved branches adjacent to the absorption-band can be traced in the original negative. Eye observations enable one to trace them much farther, for the tips are exceedingly faint, and the light is not very actinic.

In order to secure accurate measurements of the dispersion in different parts of the spectrum I have made use of two different methods.

The photographs were not very suitable owing to the fact that during the exposure the density of the vapour varied somewhat, producing a slight blurring of the image. Measurements of the deviation of the continuous spectrum were accordingly made with a filar micrometer, an illuminated scale giving the wave-lengths at the points where readings were taken. Several minutes were necessary, however, to 
get anything like a satisfactory set of readings, and changes occurred even during that short period, as was at once apparent on repeating observations. I accordingly adopted the following method.

The telescope of the spectrometer was removed and a telescope-objective of nearly two metres focus put in its place. In the focal plane of this lens a plate of glass was firmly mounted, on which a horizontal diamond scratch had been ruled. This diamond scratch was brought accurately into coincidence with the narrow contintous-spectrum image in its undeviated position. An eyepiece mounted behind the plate enabled the spectrum to be observed. When the dispersion-tube was in good condition, and the deviated branches of the spectrum appeared sharp and steady, their positions on the plate were marked with a writing diamond, the line being drawn along the middle of the spectrum, which was only about a millimetre wide on the plate. The dispersion-tube was then removed and various metals fed to the are, the bright lines being marked on the plate with the diamond. In this way a scale of wave-lengths was secured. For the determination of refractive indices we require, however, the angular deviation of the different rays. This can be determined if we know the focal length of the lens forming the image of the spectrum on the slit of the spectrometer, and the actual deviations measured in millimetres of the images of the first slit formed by light of varions wave-length at this point. 'To secure a record of this a glass plate, ruled with half-millimetre lines, was placed over the slit of the spectrometer, and the position of the lines on the glass plate in the focus of the large lens recorded with the writing diamond. This scale enabled the recorded deviations to be reduced to the actual deviations as they existed on the slit of the instrument.

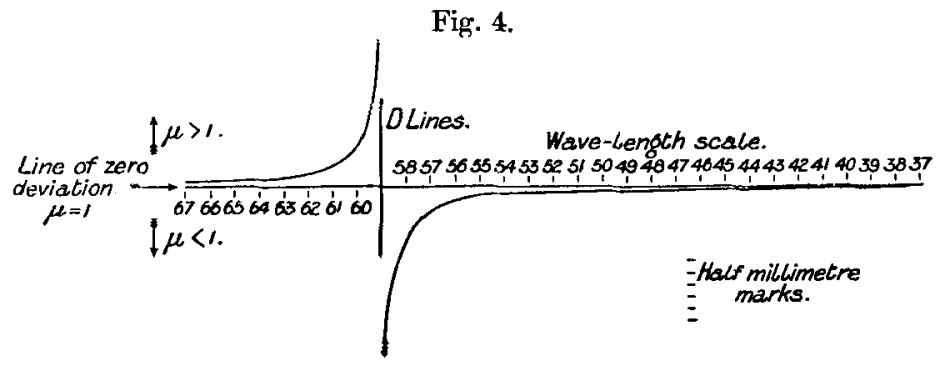

The dispersion curve obtained in this manner, with the balf-millimetre marks, is reproduced on the same scale as the original in fig. 4 , the prismatic spectrum having been 
converted into a normal spectrum. It will be seen that for all wave-lengths shorter than those of the $D$ lines, the refractive index is less than one, while for the rest of the spectrum it is greater than one. This is the only case that I know of in which we have a medium beautifully transparent even in considerable thicknesses, in which light travels faster than in a vacuum.

To determine the refractive indices we require the angle of the sodium prism, and here we encounter a grave difficulty, for it appears to be quite impossible to determine this directly. As I have said before, we are dealing with the optical equivalent of a prism, namely, a non-homogeneous medium, in which the vertical wave-front is retarded or accelerated in a progressively increasing amount as we pass from its upper to its lower edge. The equivalent prism appears from experiment to be bounded by concave instead of plane surfaces; in other words, the effective angle is greater near the bottom of the tube than at the top. This can be shown by screening off different levels.

The lower part of the tube acting alone gives a much greater deviation than the upper. In practice I have found it best to screen off the upper and lower portions, utilizing only the light which passes in the median plane, where the change in density appears to be quite uniform, this method baving been used in preparing the dispersion curve alluded to above. The effertive angle lies probably somewhere between $90^{\circ}$ and $130^{\circ}$, but even this estimate is mere guesswork.

If a single observation could be made with a vapour prism of known angle, in which we could be sure that the density was uniform, quantitative values could be assigned to determinations made with the dispersion-tubes. I have accordingly tried in every way possible to obtain a prism fulfilling the required conditions. These endeavours have thus far met with only partial success, but an account of the devices tried may be of value to others working along similar lines.

Glass and mica are so quickly atitacked by the sodium vapour, that the use of these substances for prism-faces seems to be out of the question. Added to this there is the difficulty of making gas-tight joints between plate-glass and metal which will stand a temperature but little below a red heat. Repeated failures to secure prisms in this way compelled me to seek some other method of giving the vapour the required form. I had observed that in glass tubes held before sodium flames the black vapour retreated before the approach of a cold obstacle. This suggested to me that it 
might he possible to do away with glass surfaces entirely, moulding the vaponr into the required prismatic form by the proximity of cold bodies. Experiments along these lines were partially successful. Two pieces of thick-walled iron tubing, the ends of which had been cut off at an angle, were introduced into a glass tube, and the sodium placed in the clear space between the bevelled ends, as is shown in fig. 5 . The ends of the tube were closed in the usual manner and the

Fig. 5.

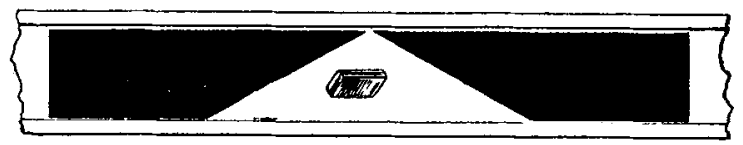

whole mounted between the collimator and telescope, in such a position that the prism formed by the sloping ends of the iron tubes stood with its refracting edge vertical. This was necessary, for in any other position the refraction due to the non-homogeneity of the vapour would have made itself felt. With a vertical slit and a vertical prism no lateral deviation could result from this cause. It was hoped that the sodium vapour formed by sudden and rapid heating of the glass tube would refuse to enter the colder iron tube, and that a prismatic form would result from the bevelled ends. The slit of the spectroscope was illuminated with monochromatic light obtained by prismatic analysis, the wave-length of which could be changed by turning the prism, which was furnished with a mirror in the manner described by Wadsworth.

On heating the tube the black vapour was seen to spread out and fill the prismatic clear space between the iron tubes, while the lateral deviation of the image of the slit, as observed in the telescope, indicated that, to a certain extent at least, the desired prismatic form had been secured. In the extreme red the deviation was very slight, but as the spectrum was advanced across the slit by slowly turning the prism the image in the telescope moved ott to one side, the deviation in this direction reaching its masimum value just before the wavelength reached that of the sodium lines. At this point the image jumped abruptly to the other side just as we should expect it to do on crossing the D lines in the spectrum, and from now on the image slowly crawled back to its undeviated position. The focal length of the telescope was $460 \mathrm{~mm}$, and the maximum deviation of the rays adjacent to the D lines on the red side, as measured by an eyepiece filar micrometer, was but $1 \mathrm{~mm}$., while the deviation in the opposite direction of the rays on the other side of the $\mathrm{D}$ lines was 
$1 \cdot 2 \mathrm{~mm}$. The angle of the prism was 130 , from which data we get the following values for the refractive index of the vapour for these two wave-lengths (relative to hydrogen): $\mu=1.0005$ and $\mu=0.9994$.

Similar results were obtained with the device shown in fig. 6, when two elliptical pieces of perforated sheet iron were used for monlding the vapour. The images formed in this case were blurred by diffraction.

Fig. 6.

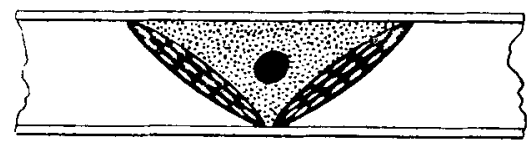

I place very little value however on these figures, for I am of the opinion that the effective angle of the sodium prism is much less than the angle between the ends of the tubes, it seeming probable that the vapour bulges out into the tubes, especially near the centre. That this is to a certain extent the case is indicated by the fact that the image of the slit is not very sharp, though this may well be caused by the varying density of the vapour. I have not yet despaired of getting a prism bounded by plates of glass, about which there can be raised no question, though the problem is a difficult one, and observations will bave to be made with great rapidity.

The deviations obtained by this method are very much less than those obtained with the dispersion-tubes, indicating either that the equivalent angle is very large in the latter case (it may be nearly $180^{\circ}$ for all we know) or that the angle of the prism formed by the iron tubes was less than the estimated value.

If we calculate the refractive indices from the data given by the curve traced with the diamond point, assuming the equivalent angle of the stratified vapour to be $130^{\circ}$, we get for the wave-lengtbs adjacent to the absorption-band the values $\mu=1.0024$ and $\mu=0.9969$.

The value given by Becquerel (1.0009) is intermediate between the values obtained by the two methods, but I feel sure that his flame must have been non-homogeneous, and his numerical results more or less inaccurate in consequence.

For the exhibition of the actual spectrum produced by a prism of sodium vapour, a long dispersion-tube with a battery of four or five prisms gives the best results. A small Bunsen burner should be used for each of the fragments of sodium, which should be at least $b^{\circ}$ or $8 \mathrm{~cm}$. apart. (This arrangement is shown in tig. 2, p. 132.) 
The coloured drawing of the spectrum, Plate II., to which I have already alluded, was made when the spectrum was obtained in this manner. A single prism gives a very pretty anomalous spectrum, but the magnificent effect produced by the battery makes the slight amount of extra trouble well worth while. If the electric arc is employed as the source of light, extreme violet will be found to occupy the position of the undeviated image of the slit. Then comes the blue, sometimes in contact with the violet and sometimes slightly separated by a fine dark line, owing to the fact that the violet light comes from the fluted carbon band of the are, which is separated from the blue by a comparatively dark region. Then comes a wide gap corresponding to light absorbed by the sodium vapour in the blue-green region (the channelled spectrum), and above this a beantiful flare of colour ranging from blue-green through grass-green to yellow. The red and orange portion of the spectrum is on the other side of or below the undeviated image, forming another brilliant flare of colour. It is separated from the violet by a wide dark band, due to the absorption in the vicinity of the $D$ lines. If the density of the vapour is increased by heating the tube to a higher temperature, the red fiure extends lower down, grows fainter, and finally fades away owing to the presence of the fluted absorption-hands in the red. The green and blue persist, however, becoming more widely separated, but finally the green disappears aimost entirely. It is best to arrange the gas-cock so that the height of the flames can be controlled without leaving the eyepiece, for it is surprising how slight a change is necessary to completely alter the general appearance of the spectrum.

The glass tube should not be allowed to cool until the experiment is at an end, otherwise it will immediately fly to pieces as soon as the flame is again applied to it.

While glass answers very well if the experiment is to be of short duration, sheet iron is much more satisfactory. Suitable tubes can be made by any tinsmith. They should be made of thin sheet-iron, and the turned-over seam hammered until a tight joint is formed. These tubes can be heated and cooled any number of times, and can be kept in operation for an hour or two, at the end of which time the sodium is generally used up, a moss-like deposit of oxide gradually filling up the tube. These tubes can be used over and over again without deterioration, and are most satisfactory in every respect. Their only fault lies in their conductivity, the sealing-wax softening and the glass plates falling off, but this can be prevented by wrapping a strip of cloth around 
each end, and wetting it from time to time. I have had one tube mate with water-jackets at each end, but it seems to have no especial advantage, and is more complicated. Porcelain tubes are quite satisfactory, but I prefer the iron on the whole.

By employing a tabe of about $5 \mathrm{~cm}$. diameter $I$ have succeeded in projecting the anomalous spectrum, but the appearance is so very inferior to that of the phenomenon when seen subjectively, that I should never think of employing the method.

No trace of any influence of the fluted absorption-bands on the dispersion has been detected.

It is a matter of some interest to know what becomes of the energy absorbed by a sodium flame, or by non-luminous sodium vapour. Most of it is undonbtedly spent in raising the temperature of the vapour; this rise of temperature I have shown experimentally by making the dispersiont-tube the chamber of a radiophone, and illuminating it with intermittent light. It was found that the radiophone sang loudly when illuminated by an intense beam of light which contained waves of the frequency of the sodium radiations, but became almost silent when these frequencies were removed.

Prismatic analysis was found to weaken the light too much : consequently a very thin tilm of cyanine was used which was quite opaque to the region of the $\mathrm{D}$ lines while transparent to the rest of the spectrum. Sunlight concentrated by a large mirror was employed for the illumination.

It has always appeared to me thinkable that the absorbing sodium ions, when in resonant vibration, might emit a certain amount of light laterally. Kayser mentions in his new work on spectroscopy an experiment made by Miller, in an attempt to detect this phenomenon if it existed. The possibility of such a lateral radiation is discussed by Drude in his Lehrbuch der Optik (p. 493), and is shown to depend on a small value of the constant $r$ in the equation of motion of an ion vibrating under the influence of a periodic electric force. For sodium vapour, $r$ is calculated to be something between 10 and 100 . If it were equal to one we should expect a lateral radiation of light, which, says Drude, has never been observed.

While experimenting with the very intense beam of sunlight in the radiophone experiment, I observed what appeared to be a lateral radiation of a clear green light by the vapour.

My first thought was that it was light scattered by the small particles of oxide which are always floating about. If this were the case, the spectrum of the light should be that of white light after transmission through sodium vapour. An 
examination with the spectroscope, however, showed that this was not the case, for the spectrum consisted of a diffused red band, a very narrow bright band in the case where the D lines occur, and a diffused hand in the green, in which flutings seemed to be present. The general appearance of the light reminds one of the fluorescence of uranium glass.

$I$ am at the present time engaged in an investigation of the spectrum of this fluorescent light, but am not yet prepared to say whether the flutings in the green are discontinuities in the emission or due to the absorption of the non-luminous vapour surrounding the fluorescent vapour. A careful photographic stndy will be necessary before this can be decided, for which investigation I am employing an iron tube provided with copper jackets at each end, through which water circulates. (Fig. 7.) The light emitted laterally is observed through a second iron tube inserted at a right angle, and provided with a glass window. The sodium is placed at the junction of the tubes, which is then raised to a red heat with a blast-lamp.

Fig. 7.

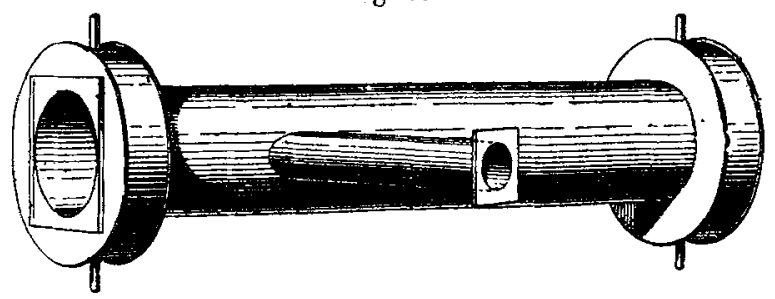

So far as I am able to judge by passing the light causing the fluorescence through colour-screens, the wave-length of the emitted light corresponds very nearly to that of the exciting vibrations. Interposing a thin cyanine screen, which removes the light in the neighbourhood of the D lines, causes no apparent diminution in the intensity of the emitted light; but the spectroscope shows that the bright band at wave-length 5890 bas disappeared. A film stained with aurantia, which removes everything below the " $b$ " group, greatly dininishes the intensity of the fluorescent light, and changes its colour to red. A solution of cuprammonium of moderate strength removes from the light all of the wavelengths which are capable of exciting the fluorescence. A more careful investigation will be necessary before deciding whether the wave-length of the emitted light is greater than that of the exciting vibrations. My impression is that the bright narrow band in the yellow is slightly on the red side 
of the D lines, but I do not feel absolutely sure of it. It will be interesting to see whether the fluorescence persists for an appreciable time after the light is cut off, which can be easily determined with an especially designed phosphoroscope, a matter which I expect to take up next.

A quantitative study of the absorption of the vapour is extremely desirable, though the experimental difficulties will be very great. As I bave already said, the width of the band at the $\mathrm{D}$ lines is often from ten to fifteen times the distance between the lines. Julius has, however, called attention to the fact that we must be on our guard against attributing the absence of light in the spectrum to absorption, when the conditions are such that the rays may have been merely turned to one side by refraction. This he believes, if I read his paper rightly, is the true explanation of the widening of the $D$ lines in the absorption-spectrum. To eliminate the possibility of this lateral deviation it is necessary, if we are working with a non-homogeneous medium, to arrange matters so that the rays of light are perpendicular to the surfaces of equal refractive index, instead of parallel, as is the case in the dispersion-tubes. One obrions method of getting round the difficulty would be to vaporize the sodium in a vacuum, thus doing away with the variations in the density, but this necessitates contact between the corrosive vapour and the glass. A better plan appeared to be to send the light through the stratified vapour in such a direction that no lateral deviation could result, a matter of some difficulty until the expedient occurred to me of using the surface of the molten metal as a reflector, the rays thus twice traversing the nonhomogeneous medium in a direction perpendicular to the equi-indicial surfaces.

A photograph of the absorption-spectrum of the vapour in the red and yellow region, obtained with a 10-foot concave grating, is reproduced on Plitte IV. fig. 12. The $\mathrm{D}$ ines were photographed just below the spectrum for comparison. The flutings in the red are well shown in this picture, which is but one of a large number that have been taken. Measurements of wave-length have not yet been made, as I feel confident of getting better plates in the near future. The fine dark lines can be found on both sides of the heavy band at the $D$ lines, and $I$ have found that they are present throughout the entire spectrum. An enlargement of a portion of fig. 12 is shown in fig. 13 (Pl. IV.) which gives a better idea of the appearance of the fluted bands.

On increasing the density of the vapour the red end of the spectrum finally disappears, and by employing a very powerful 
light in combination with the method above alluded to of reflocting the light from the mirror of molten sodium, I find that the spectruin consists of two exceedingly brilliant narrow green bands with a narrow dark line between them, and a band in the violet which is partially filled with flutings. This spectrum I have observed but once, and have given it no careful study as yet.

The flutings in the green-blue portion of the spectrum are similar in appearance to those in the red, but they make their appearance when the vapour is less dense, this part of the spectrum being blotted out entirely when the red bands are at their best.

I have not yet investigated the question of whether an increase in the thickness of the layer of vapour is the equivalent of increasing its density, but I feel quite sure that such is not the case. There remains too the question of pressure. All of the experiments that $I$ have made thus far have been at atmospheric pressure, the variations in density being in all probability merely dilution of the metallic vapour with hydrogen.

Sodium vapour possesses another property which requires further investigation, namely, the power of emitting light in virtue of temperature alone. This was first observed by Evershed *. Iodine and a number of other substances behave in a similar manuer, the temperature necessary to produce the luminous radiations being but little above a red heat. On repeating Evershed's experiment with iodine, my first im। ression was that anomalcus dispersion might be at the bottom of it. 'The vapour was heated in a tube, and the purple luminosity or glow hung close to the inner wail, which was red hot. It seemed quite possible that the phenomenon might be a mirage effect, the rays of suitable wave-length coming from the red-hot wall moring in eurved paths through the non-homogeneous vapour. To test this I devised a method by which this possibility was entirely eliminated, and found that the glow was more brilliant than ever. A spiral of platinum wire mounted in a small flask in which iodine was vaporized was raised to a bright red heat by an electric current. The convection current of iodine which rose through the spiral was luminous to a height of about $2 \mathrm{~cm}$. above the spiral, waving from side to side in the form of a reddish-purple flame. Focussing the image of an electric arc on the spiral increased its luminosity ten-fold, without, bowever, altering the intensity of the glowing vapour. Probably the luminescence

* Phil. Mag. [0] xxxix. p. 460 (May 1895). 
of sodium could be shown in the same way, though the one experiment that I tried with it gave negative results.

I expect in the near future to investigate all of the questions which $I$ have touched upon in the latter part of this paper, for it appears to me that, with proper coaxing, sodium vapour may furnish us with much information regarding molecular dynamics. The results obtained up to the present time are only a small heginning, but appear to indicate that in sodium vapour we have a medium which approaches more nearly to the ideal absorbing medium, with but a single natural period of vibration, thim any substance heretofore investigated.

Of course I am speaking here only with reference to the natural vibration which appears to influence the dispersion. Strictly speaking there are two natural periods of course, which infuence the velocity of the light in the medium, but when the medium is very dense the condition certainly approaches very nearly to that of a single period medium. As 1 have said before, the fluted absorption-bands are without influence on the dispersion, at least their influence is too slight to be detected by the methods that have been emplojed thus far.

XIV. Note on Capillarity Constants of Crystal Faces. By Harold Hilín *.

N page 520 of the 34 th volume of the Zeitschr. f. Kryst. u. Min. G. Wulff gives a very interesting theorem; he says:- "Nelımen wir an, dass wir ein Polyëder mit gegebenen Flächenrichtungen, welche durch die Normalen $n_{1}, n_{2}, n_{3} \ldots$ bestimmt werden, gefunden haben, welches der Bedingung des Minimums der Oberflächenenergie bei gegebenem Volumen Genüge leistet. Es ist einleuchtend, dass alle Polyëder welche dem letzteren ähnlich sind und nur durch ihre Volumen sich von einander unterscheiden, ebenfalls dieser Bedingung Genüge leisten werden. Alle dieser Polyëder werden einen Krystall in verschiedenen Wachstumsstadien vorstellen, wobei es klar ist, dass der Wachstumsanfang mit dem letzten Centrum der Aehnlichkeit der ganzen erhaltenen Polyëderreihe zusammenfallen muss. Auf solche Weise kann man die Oberfläche eines dieser Polyëder folgendermassen ausdrucken : $p\left(n_{1}{ }^{2}+n_{2}{ }^{2}+n_{3}{ }^{3}+\ldots\right)$, wo $p$ eine Constant ist, und die Oberffïchenenergie $\mathbf{E}$ bei den Capillaritätsconstanten $k_{1}, k_{2}, k_{3}, \ldots$ auf den verschiedenem Flächen

$$
\mathrm{E}=p\left(k_{1} n_{1}{ }^{2}+k_{2} n_{2}{ }^{2}+k_{3} n_{3}^{2}+\ldots\right)
$$

* Communicated by the Author. 


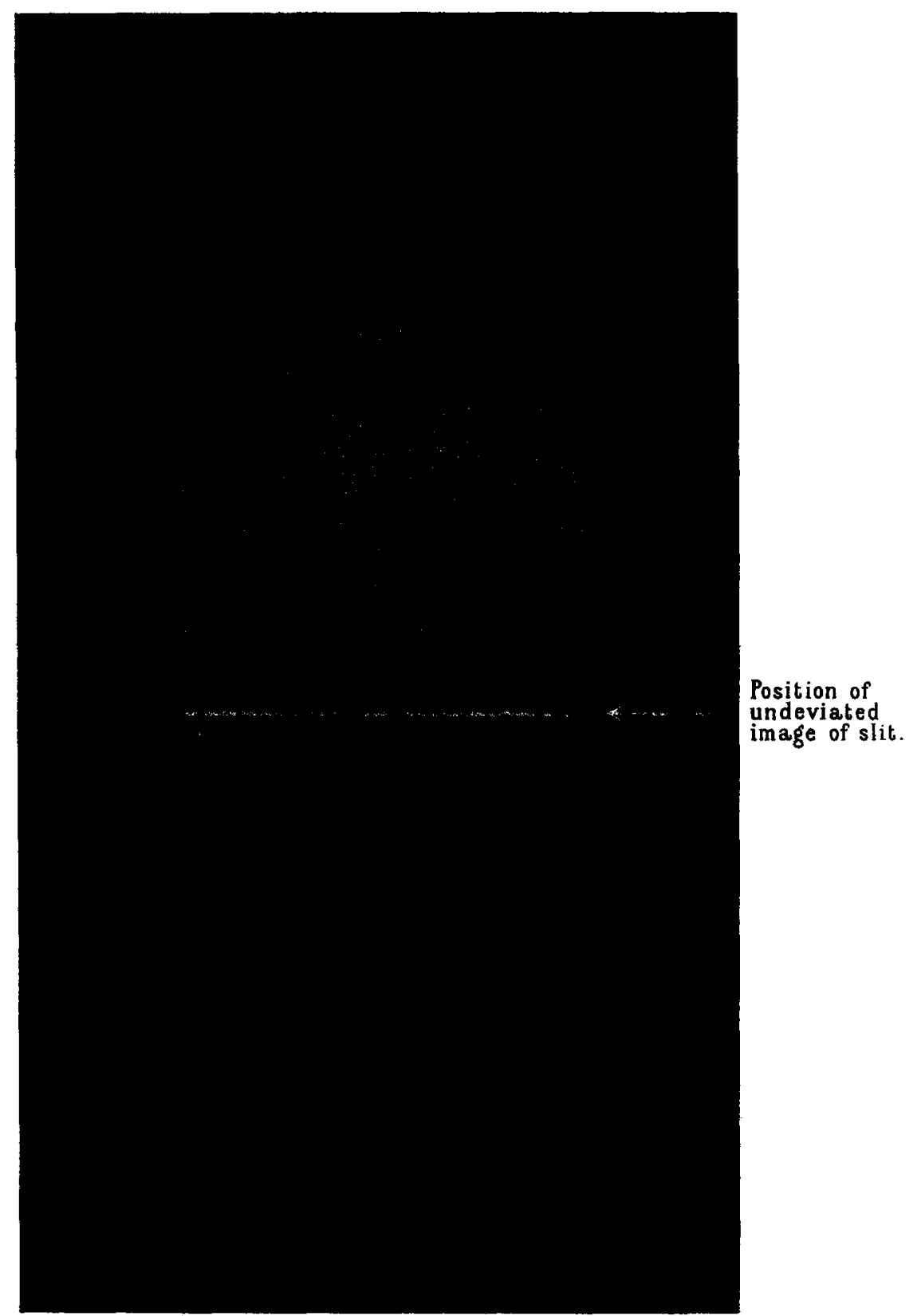

West, Newiman chromo.

Anomalous Spectrum produced by Prism of Sodium Vapour. 
Phil. Mug., S. 6, Vol. 3, Pl. IIT.

Fro. 8

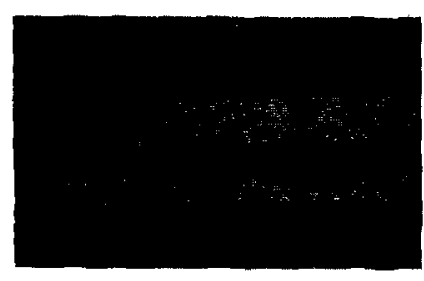

Fig. 9.

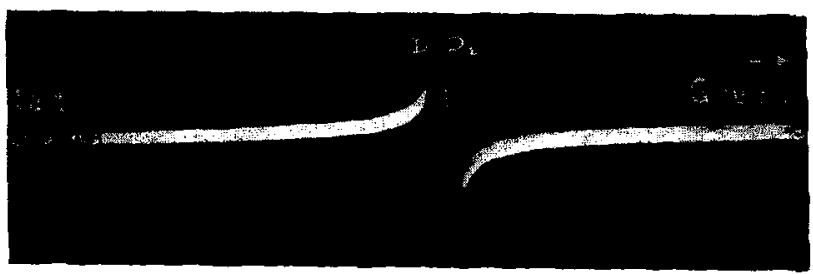

Fıg. 11.

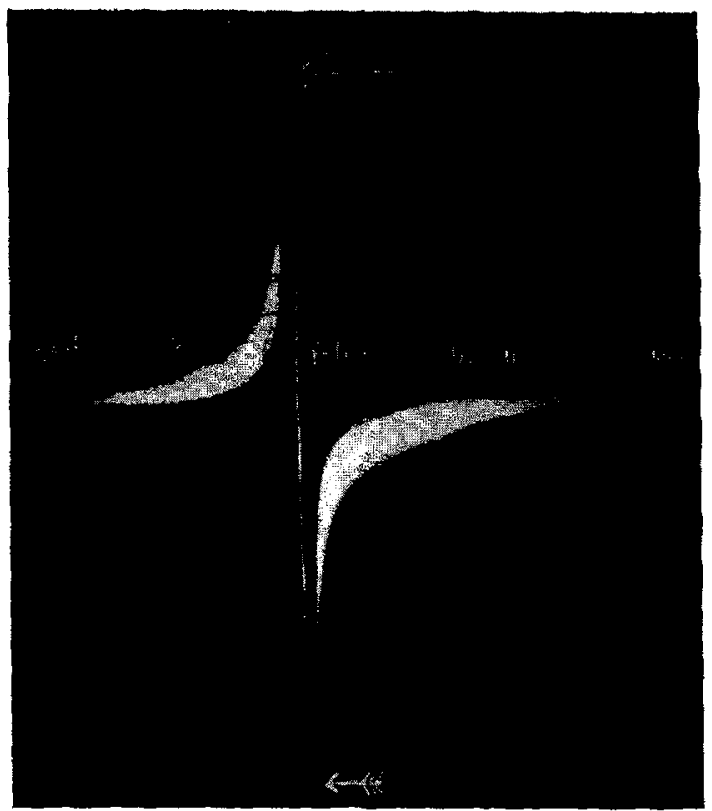




\section{Phil. May., S. 6, Vol. 3, Pl. IV.}
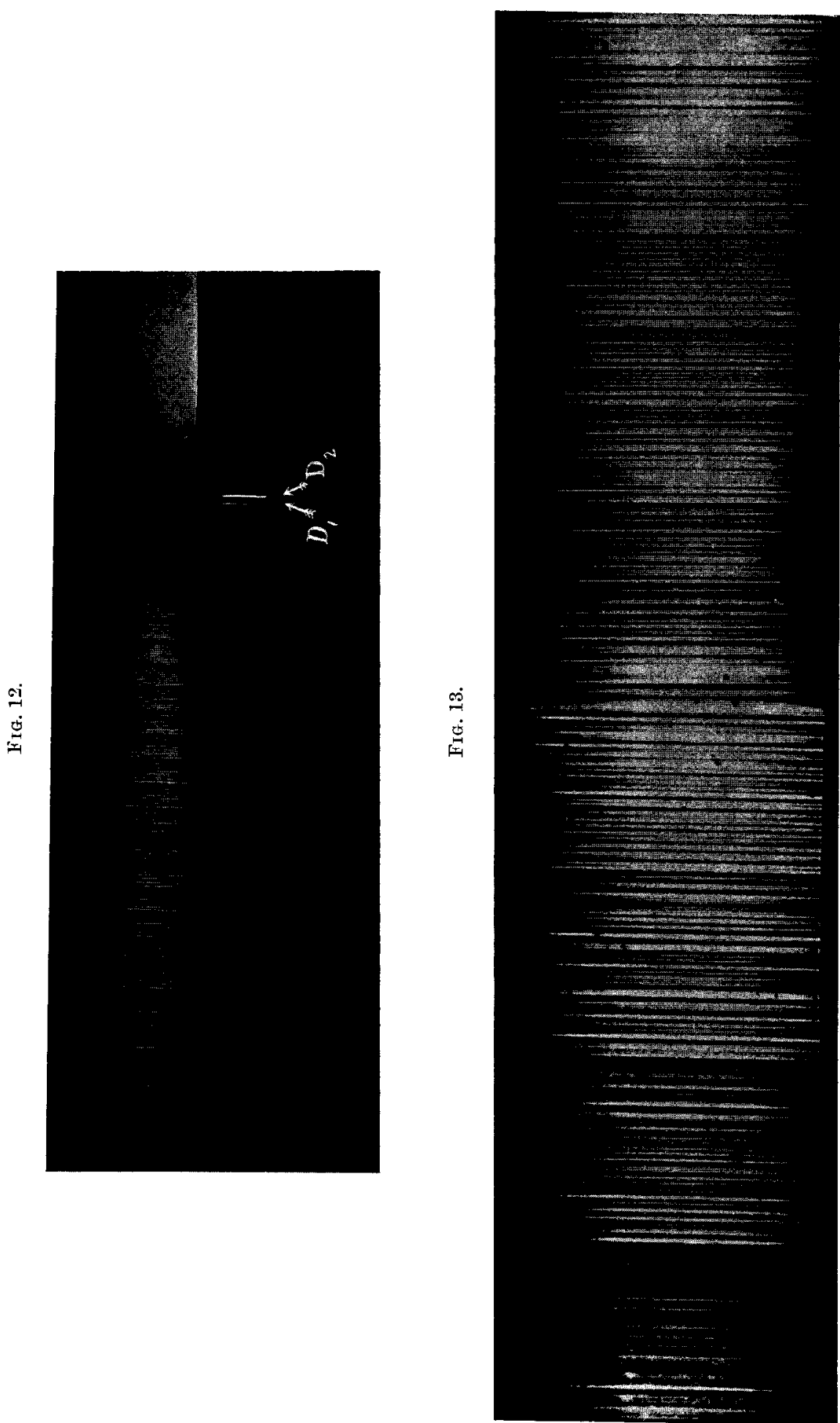